\title{
Synthesis of novel active ester ether resin and its effects on positive thermal CTP plates
}

\author{
SHA XuZheng, PANG YuLian \& ZOU YingQuan* \\ College of Chemistry, Beijing Normal University, Beijing 100875, China
}

Received June 11, 2012; accepted August 6, 2012; published online April 1, 2013

\begin{abstract}
A novel active ester ether resin, consisting of $p$-hydroxyphenylacetic acid and a mixed-phenol novolac resin, was produced by the addition of ethyl vinyl ether to a carbonyl novolac resin. This study investigated the conditions of its synthesis and characterized its structure using infrared and ${ }^{1} \mathrm{H}$ NMR spectroscopies, and gel-permeation chromatography. Its solubility in alkaline solution differed greatly before and after decomposition. The results of thermal gravimetric analysis confirmed the formation of an active ester ether resin of $p$-hydroxyphenylacetic acid and novolac resin. The resin had acidolysis activity at room temperature and pyrolysis activity at high temperatures $\left(140-200^{\circ} \mathrm{C}\right)$ and could be used in the imaging layer of a positive thermal plate for computer-to-plate processes as an inhibitor/accelerator to improve its alcohol resistance, alkali resistance, sensitivity, lattice point reducibility, and developing tolerance.
\end{abstract}

active ester ether resin, inhibitor/accelerator, positive thermal CTP imaging, positive thermal CTP plate Citation: $\quad$ Sha X Z, Pang Y L, Zou Y Q. Synthesis of novel active ester ether resin and its effects on positive thermal CTP plates. Chin Sci Bull, 2013, 58: 1536-
1542, doi: 10.1007/s11434-013-5797-Z

With the rapid development and application of digital technology in the printing industry, the protocol of positive thermal imaging with an 830-nm infrared (IR) laser has become mainstream because it is easy to use, has high sensitivity, and does not involve pre-baking.

As shown in Figure 1, the thermal imaging layer is important in plates for positive thermal computer-to-plate (CTP) imaging. Early positive thermal CTP imaging layers were generally composed of an IR dye (photothermal conversion reagent), a background dye, an acid-producing reagent, and a film-forming resin. In the imaging process, the IR and background dyes commonly act as physical inhibitors. Their alkaline amino groups build a compact hydrogen-bonded network by associating with phenolic hydroxyl groups in the film-forming resin. This results in a large decline in the imaging layer's dissolution velocity in alkaline developers. When it is scanned with an 830-nm laser, the temperature of the exposed area of the plate rises to nearly

\footnotetext{
*Corresponding author (email: zouyq@263.net)
}

$300^{\circ} \mathrm{C}[1]$ as the 830 -nm laser's energy is converted into heat energy by the IR dye during exposure. As a result, the hydrogen bonds are completely destroyed. The exposed area then dissolves quickly in the alkaline developer and the anticipated pattern is formed [2]. The drawbacks of plates made from systems with inhibiting dyes are that they need to be post-baked, and they have poor chemical resistance, a small press run, and a short shelf life.

Some new types of inhibitors/accelerators have been reported in the literature. Yu et al. [3-5] synthesized a series of ester ether resins and small-molecular active ester ether compounds, which greatly improved the sensitivity, alcohol resistance, and developing tolerance of plates. However, their hydroxyl groups reacted with acid anhydrides, forming ester bonds which did not decompose at the time of thermal imaging. This resulted in poor imaging sensitivity. Feng et al. [6] produced a type of novolac resin by the addition of isocyanate groups to hydroxyl groups, but its pyrolysis activity was not high. Yeh et al. [7] synthesized a copolymer of a methacrylic acid monomer containing metal(II) (zinc, 


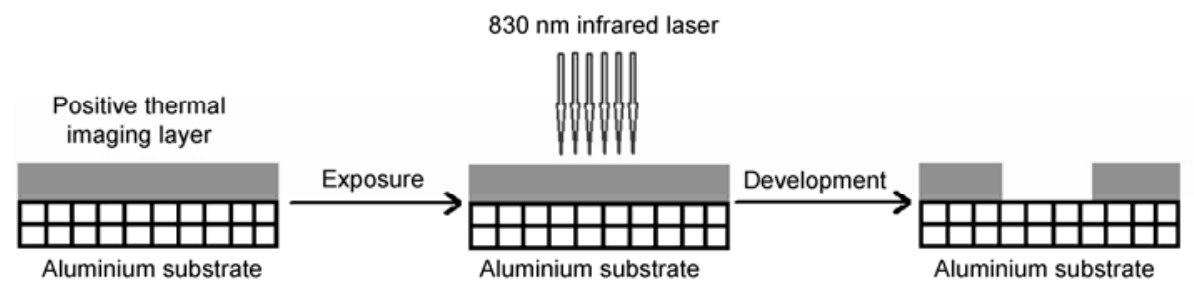

Figure 1 Imaging mechanism of positive thermal CTP plate.

magnesium, or copper) ions, and its derivatives with other free radicals, as inhibitors/accelerators. They were only able to improve the abrasion resistance of the plate. Li et al. [8] synthesized a form of poly(methyl acrylate) with acidolysis activity at room temperature, but this type of inhibitor/accelerator had a low softening point (below $85^{\circ} \mathrm{C}$ ), resulting in soft films. We [9] synthesized an inhibiting/accelerating phenolic etherified resin. It had pyrolysis activity at high temperatures $\left(150-220^{\circ} \mathrm{C}\right)$, and improved sensitivity of the imaging layer. However, it is a phenolic resins, so it has low solubility in alkaline media, which leads to insufficient solubility differences between the exposed and unexposed areas in alkaline developers.

We carried out further research to improve the sensitivity of positive thermal CTP plates and enhance the solubility differential between the exposed and unexposed areas in alkaline developers. In this paper, we report a novel active ester ether resin consisting of $p$-hydroxyphenylacetic acid and a mixed-phenol novolac resin (DF2), which is based on a novel $p$-hydroxyphenylacetic acid/mixed-phenol novolac resin (DF1), with good solubility in alkaline media. It has acidolysis activity at room temperature and pyrolysis activity at high temperatures $\left(140-200^{\circ} \mathrm{C}\right)$, and gives a high solubility differential between the exposed and unexposed areas in alkaline developers. Consequently, it has good inhibiting/accelerating properties in positive thermal CTP imaging. More importantly, because of the novel structure, it improves the alcohol resistance, alkali resistance, sensitivity, lattice point reducibility, and developing tolerance of a positive thermal CTP plate. Moreover, it is not necessary for the plate to be pre-/post-baked.

\section{Materials and methods}

\subsection{Materials}

BTB-225 novolac resin was purchased from Weihai Tiancheng Chemical Co., Ltd. BX-60 novolac resin was purchased from Benxi Rst Chemical Co., Ltd. Victoria blue was imported from Japan. IR cyanine dye (ICD) [10] and 2, 4,6-tris(trichloromethyl)-1,3,5-triazine (TTCMT) were purchased from Hubei YuKui Chemical Co., Ltd. The developer was purchased from Taixing City Orient Industrial Company. Ethylene glycol monomethyl ether, propylene glycol monomethyl ether (PGMME), and other reagents were purchased from Beijing Chemical Plant.

\subsection{Instruments}

Aluminum substrate: PF-I; spin coater: SC-I (Taixing City Orient Industrial Company); plate setter: Screen 8600S plate setter (Dainippon Screen Manufacturing Co., Ltd.); IR spectrometer: NICOLET 380 FTIR (Thermo Electron Corporation); gel-permeation chromatography: PL-GPC50 column (United Kingdom Polymer Laboratories Ltd.); digital display micro-melting point meter: X-4 (Beijing Tech. Instrument Co., Ltd.); metallographic microscope: HJ1 (Chong Qing Optical \& Electrical Instrument Co., Ltd.); X-Rite iCplate II: (X-Rite Inc.); thermal gravimetric analyzer: Mettler-Toledo TGA/DSC 1/1100 (Switzerland).

\subsection{Synthesis}

(1) Synthesis of DF1. The synthetic routes for DF1 are shown in Figure 2. A four-necked flask equipped with an agitator, condenser, constant-pressure addition funnel, and mercury-in-glass thermometer was charged with $p$ hydroxyphenylacetic acid (16.72 g, $0.11 \mathrm{~mol}), p$-cresol $(7.56 \mathrm{~g}, 0.07 \mathrm{~mol}), m$-cresol $(4.32 \mathrm{~g}, 0.04 \mathrm{~mol})$, and formaldehyde aqueous solution $(17.0 \mathrm{~g}, 0.21 \mathrm{~mol}, 37 \%)$. The reaction mixture was heated up to $80{ }^{\circ} \mathrm{C}$, and oxalic acid $(0.60 \mathrm{~g}$, $4.76 \mathrm{mmol}$ ) was added. Heating was continued until the reaction mixture reached a temperature of $100-110^{\circ} \mathrm{C}$. The temperature was maintained for $5 \mathrm{~h}$, and the unreacted materials were removed by vacuum distillation from $110^{\circ} \mathrm{C}$ to $200^{\circ} \mathrm{C}$ for $2 \mathrm{~h}$. Then the residue was allowed to cool to room temperature. The product, DF1 (26.3 g), was precipitated as a yellowish solid [11]. IR $\left(\mathrm{KBr} ; \mathrm{cm}^{-1}\right): 3226.9(\mathrm{~s}$, -OH), 3011.2 (s), 2952.9 (s), 1705.3 (s, C=O), 1611.5 (m), $1507.1(\mathrm{~m}), 1481.0(\mathrm{~s}), 1440.8(\mathrm{~s}), 1405.0(\mathrm{~s}), 1275.6(\mathrm{~s})$, $1209.1(\mathrm{~s}), 1145.9(\mathrm{~s}), 913.9(\mathrm{~m}), 865.4(\mathrm{~m}), 803.4(\mathrm{~s})$, $617.9(\mathrm{~s}), 585.7(\mathrm{~m}) ;{ }^{1} \mathrm{H}$ NMR $\left(500 \mathrm{MHz}\right.$, acetone- $\left.d_{6}\right): 11.1$ $(\mathrm{m}, 1.0 \mathrm{H},-\mathrm{COOH}), 6.8-7.0(\mathrm{~m}, 5.8 \mathrm{H}), 5.3-5.5(\mathrm{~m}, 2.6 \mathrm{H})$, 3.9-4.1 ( $\mathrm{m}, 2.2 \mathrm{H}$, methylene between two benzenes),

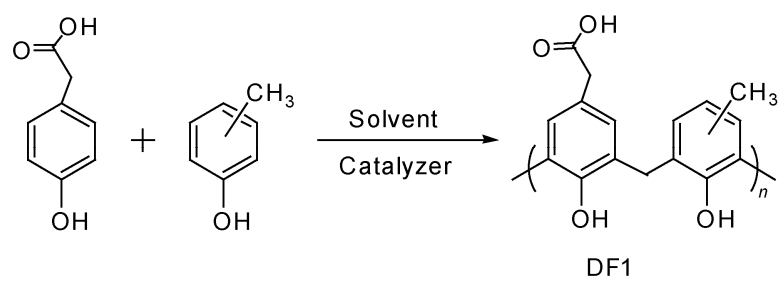

Figure 2 The synthetic routes for DF1. 
2.6-2.8 (m, 3.2H), 1.6-1.8 (m, 2.6H).

(2) Synthesis of DF2. The synthetic routes for DF2 are shown in Figure 3. A four-necked flask equipped with an agitator, condenser, constant-pressure addition funnel, and mercury-in-glass thermometer was charged with DF1 (14.3 g), sulfamic acid $(1.00 \mathrm{~g}, 0.01 \mathrm{~mol})$, dichloromethane (30 $\mathrm{mL})$, and acetone $(30 \mathrm{~mL})$. Vinyl ethyl ether $(25 \mathrm{~mL}, 0.26$ mol) was added dropwise to the solution, with stirring, at room temperature for $30 \mathrm{~min}$. The reaction mixture was then stirred at $35^{\circ} \mathrm{C}$ or $4 \mathrm{~h}$. After vacuum distillation, the residue was poured into deionized water. The precipitate was collected by filtration and dried under vacuum at $60{ }^{\circ} \mathrm{C}$. DF2 was obtained (20.6 g) as a yellow powder [12]. IR $\left(\mathrm{KBr} ; \mathrm{cm}^{-1}\right)$ : $2962.4(\mathrm{~s}), 2937$ (s), 2894.5 (s), 1728.4 (s, $\mathrm{C}=\mathrm{O}), 1471.2$ (s), 1444.2 (m), 1388.2 (s), 1243.0 (s), 1155.8 (s), 1033.2 (m, O-R), 993.2 (s), 967.2 (m), $851.8(\mathrm{~m}) ;{ }^{1} \mathrm{H}$ NMR $\left(500 \mathrm{MHz}\right.$, acetone- $\left.d_{6}\right)$ : 7.6-7.8 $(\mathrm{m}, 1.0 \mathrm{H}), 7.2-7.4$ $(\mathrm{m}, 1.6 \mathrm{H}), 5.2-5.4(\mathrm{~m}, 2.4 \mathrm{H}$, the characteristic peak of the active ester), $3.6-3.8(\mathrm{~m}, 6.1 \mathrm{H}$, the characteristic peak of the active ether), 3.4-3.6 (m, 7.9H), 3.2-3.4 (m, 4.5H), 1.9-2.2 $(\mathrm{m}, 8.9 \mathrm{H}), 1.1-1.6(\mathrm{~m}, 8.4 \mathrm{H})$.

\subsection{Detection}

(1) Preparation of positive thermal CTP emulsion and positive thermal CTP plate samples. Victoria blue, TTCMT, 830-nm ICD, BTB-225, BX-60, and DF2 were dissolved in

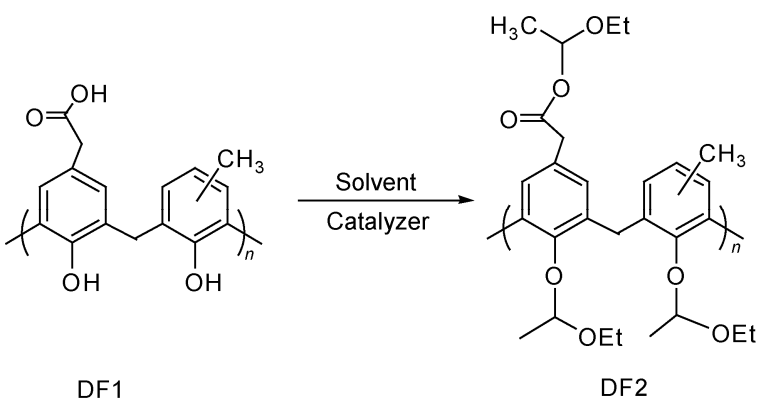

Figure 3 The synthetic routes for DF2. a mixture of butanone and PGMME (butanone:PGMME = 6:4) and filtered using a 6A filter to obtain an emulsion $(w / w=10 \%)[13]$.

The resulting five types of thermal emulsions were respectively spin-coated on aluminum substrates $(500 \mathrm{~mm} \times$ $450 \mathrm{~mm}$ ) to produce positive thermal CTP plate samples. The plate samples were dried in circulating hot air at $100 \pm$ $2{ }^{\circ} \mathrm{C}$ for $10 \mathrm{~min}$. The mass of the dry emulsion layer was $1.60-1.65 \mathrm{~g} \mathrm{~m}^{-2}$ [14].

As shown in Table 2, as the amount of DF2 increased, the amount of BTB-225, whose properties hardly influenced the performance of the imaging layer, was modulated to keep the mass percentage of the emulsion at $10 \%$. The amounts of the other ingredients were kept constant.

(2) Detection of effect of DF2 on positive thermal CTP plate. Plate setter (plate-making energy: 90-130 mJ); developer: Positive Thermal CTP-II, produced by Taixing City Orient Industrial Company; developing machine: GRAFMAC W-PTP-CDN, brush speed: 30-50 r min ${ }^{-1}$, development temperature: $23-27^{\circ} \mathrm{C}$, development time: 20-35 s; lattice point meter: X-Rite iCPlate2; each measurement was repeated three times, and the lattice point value is the average of the three values obtained by measuring each lattice point three times.

The detection methods for the imaging layer mass, alcohol resistance, and alkali resistance have already been reported by our group [15]. The sensitivity was determined by observing whether or not the bottom color remained when one drop of acetone was dropped on the blank part after exposure and developing. The lattice point reducibility was obtained by measuring the $50 \%$ lattice point after exposure with $85 \%$ laser energy and developing. The lattice point measurements were repeated three times for each sample.

\section{Results and discussion}

\subsection{Synthesis and characterization}

(1) Synthesis of DF2. The product should be insoluble in

Table 1 Gel-permeation chromatography data for DF1 and DF2

\begin{tabular}{ccccccccc}
\hline Types & Retention time $(\min )$ & $M_{\mathrm{n}}$ & $M_{\mathrm{w}}$ & $M_{\mathrm{z}}$ & $M_{\mathrm{z}+1}$ & $M_{\mathrm{w}} / M_{\mathrm{n}}$ & $M_{\mathrm{z}} / M_{\mathrm{w}}$ & Area $(\%)$ \\
\hline DF1 & 11.438 & 1176 & 4318 & 14077 & 30736 & 3.67314 & 3.25969 & 100 \\
DF2 & 12.134 & 1426 & 4913 & 14587 & 32091 & 3.44523 & 2.96913 \\
\hline
\end{tabular}

Table 2 Plate types and percentages of emulsion ingredients

\begin{tabular}{|c|c|c|c|c|c|c|}
\hline Types & VB (\%) & TTCMT (\%) & $\operatorname{ICD}(\%)$ & BTB-225 (\%) & BX-60 (\%) & DF2 (\%) \\
\hline CTP-1 & 2.0 & 2.0 & 2.0 & 73 & 15 & 5 \\
\hline CTP-2 & 2.0 & 2.0 & 2.0 & 68 & 15 & 10 \\
\hline CTP-4 & 2.0 & 2.0 & 2.0 & 58 & 15 & 20 \\
\hline CTP-5 & 2.0 & 2.0 & 2.0 & 53 & 15 & 25 \\
\hline
\end{tabular}


alkali, so it is desirable for it to have no hydroxyl groups. This means it should be produced by complete etherification. We therefore developed an effective formula for testing the rate of etherification to monitor the progress of the reaction: $X=\left(h_{1} \times W_{1}^{1 / 2}\right) /\left(h_{2} \times W_{2}^{1 / 2}\right)$, where $\left(h_{1} \times W_{1}{ }^{1 / 2}\right)$ is the product of the phenolic hydroxyl peak height and the width at half-peak height, and $\left(h_{2} \times W_{2}^{1 / 2}\right)$ is the product of the peak height and the width at half-peak height of $\mathrm{C}=\mathrm{C}$ in the benzene ring, at $1610-1630 \mathrm{~cm}^{-1}$. The smaller the value of $X$ is, the greater the etherification of hydroxyl groups in the addition reaction with vinyl ether.

Five aspects of the DF2 synthesis can be investigated: solvent, catalyst, temperature, reaction time, and the molar ratio of vinyl ether to hydroxyl groups.

(2) Effect of solvent on the reaction. An acidic catalyst can make the reaction of DF1 and vinyl ethyl ether reversible, but the reverse reaction can be avoided by using a solvent in which DF2 is diffluent. The polarity of DF2 is much weaker than that of DF1, so a weakly polar solvent favored the reaction. The solubilities of the other reaction components in the solvent must also be taken into consideration, especially that of the catalyst. We found that a mixture of dichloromethane and acetone in a 1:1 volume ratio favored the synthesis of DF2 [9].

(3) Effect of catalyst on the reaction. Theoretically, strong acid catalysts are better in the reaction of phenolic hydroxyl and vinyl ether groups than weak acid catalysts. However, strong acids also promote side reactions and selfpolymerization of vinyl ether. For example, a phenolic hydroxyl and vinyl ether barely react with each other when an inorganic acid (e.g., phosphoric acid) catalyst is used. Appropriate catalysts show high catalytic efficiency and avoid undesired side reactions.

Gallucci et al. [16] reported that trifluoroacetic acid could catalyze the reaction between hydroxyl groups and vinyl ethers as an effective hydroxyl etherification catalyst, but it is too expensive for use in large-scale production. We found that $p$-toluenesulfonamide was a satisfactory catalyst, but it was difficult to remove from the production mixture because of its low solubility in water. This would influence the stability and imaging performance of DF2. Consequently, $p$-toluene sulfonamide was not chosen. Wang et al. [17] obtained good results using sulfamic acid in a 1:20 molar ratio to hydroxyl groups. Importantly, sulfamic acid is soluble in water and cheap, so sulfamic acid was suitable for use as a catalyst in the synthesis of DF2.

(4) Effect of temperature on the reaction. Based on the above discussion, a mixture of dichloromethane and acetone in a 1:1 volume ratio was used as the solvent and sulfamic acid was used as the catalyst in the experiments. The value of $X$ was small when the reaction temperature was below $35^{\circ} \mathrm{C}$, and the value of $X$ increased with increasing temperature above $36^{\circ} \mathrm{C}$. This meant that etherification of hydroxyl group was difficult when the reaction temperature was higher than $35^{\circ} \mathrm{C}$. A possible reason might be that vinyl ethyl ether vaporizes and is inclined to self-polymerize above $36^{\circ} \mathrm{C}$. The optimum temperature for DF2 synthesis was therefore $35 \pm 1^{\circ} \mathrm{C}$.

(5) Effect of reaction time. Using the chosen solvent and catalyst and a temperature of $35 \pm 1{ }^{\circ} \mathrm{C}$, the value of $X$ for different reaction times was studied semi-quantitatively.

As shown in Figure 4, the value of $X$ gradually decreased with increasing reaction time. This meant increased conversion of hydroxyl groups. When the reaction time exceeded $4 \mathrm{~h}$, the value of $X$ dropped to zero. This meant that the reaction was completed and the hydroxyl groups in DF1 were completely protected. It was concluded that a reaction time of $4 \mathrm{~h}$ was appropriate for DF2 synthesis.

(6) Effect of molar ratios of vinyl ether and hydroxyl groups on the reaction. Using the chosen solvent and catalyst, a temperature of $35 \pm 1^{\circ} \mathrm{C}$ and a reaction time of $4 \mathrm{~h}$, the value of $X$ was determined for different molar ratios of vinyl ether to hydroxyl groups.

As shown in Figure 5, with increasing molar ratio of vinyl ether to hydroxyl, the value of $X$ gradually decreased. When the ratio of hydroxyl groups to vinyl was 1.75 , the value of $X$ dropped to zero. It was concluded that the appropriate molar ratio of vinyl ether to hydroxyl group in the synthesis is around 1.75:1.

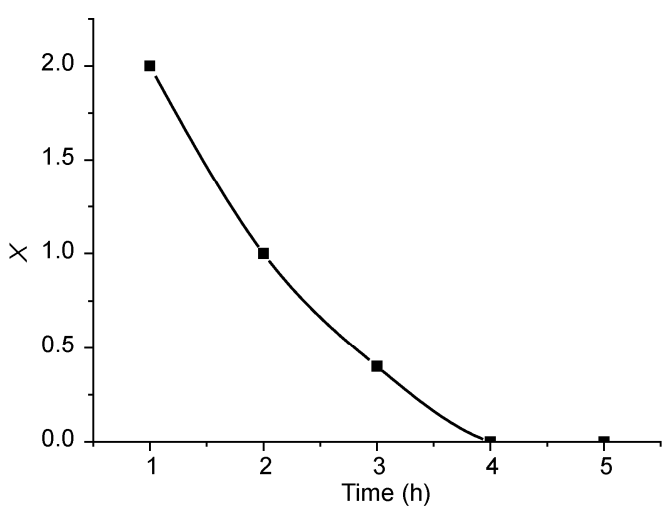

Figure 4 Relationship between the value of $X$ and reaction time.

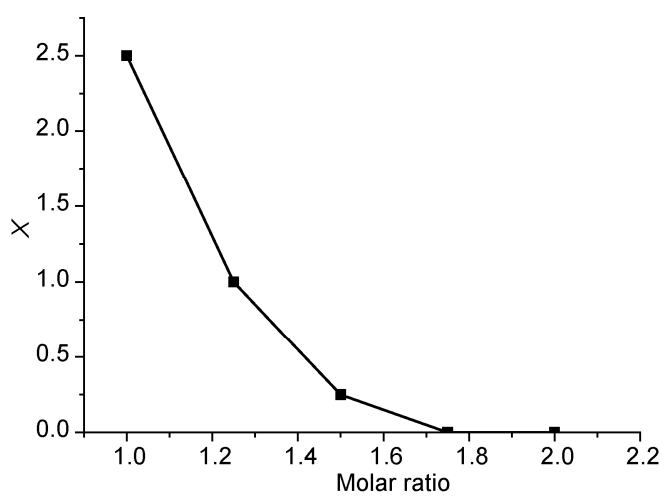

Figure 5 Relationship between the value of $X$ and the molar ratio of hydroxyl groups to vinyl ether. 


\subsection{Characterization}

(1) Melting ranges and solubilities of DF1 and DF2. The melting ranges of DF1 and DF2 were measured using an $\mathrm{X}-4$ melting point measurement apparatus with a heating rate increase of $2^{\circ} \mathrm{C} \mathrm{min}$. The solubility was determined at $20 \pm 1^{\circ} \mathrm{C}$.

As shown in Table 3, the melting range of DF2 is lower than that of DF1. The reason might be that new ether bonds weakened the intermolecular hydrogen-bonds after the etherification reaction. The solubilities of DF1 and DF2 in $\mathrm{NaOH}(1 \%)$ solution differ greatly. This is crucial for an ester ether resin to act as a good inhibitor/accelerator. The solubility of DF2 in the organic solvents used was lower than that of DF1. This means that DF2 has good chemical resistance.

(2) Thermogravimetric analysis of DF2. Stability is crucial for inhibitors/accelerators. For a positive thermal CTP plate, the decomposition temperature of the inhibitor/accelerator should not be too high or too low. If it decomposes below $100^{\circ} \mathrm{C}$, accompanied by drying of the CTP plate, a serious loss of the imaging layer occurs. If it decomposes above $250^{\circ} \mathrm{C}$, the CTP plate would have low sensitivity. The temperature range was designed to be 50$700^{\circ} \mathrm{C}$, and the heating rate was $10^{\circ} \mathrm{C} \mathrm{min}^{-1}$.

As shown in Figure 6, DF2 decomposition started at $140^{\circ} \mathrm{C}$ and ended at about $204^{\circ} \mathrm{C}$ in the first phase. The decomposition temperature range is wide because the ester ether resin's low heat conductivity produces a large internal temperature gradient, which means that the temperature of the interior reaches the decomposition temperature long after the surface does. The decomposition was believed to be caused by hydroxyl group deprotection, during which DF2 decomposed completely into DF1 and vinyl ethyl ether.

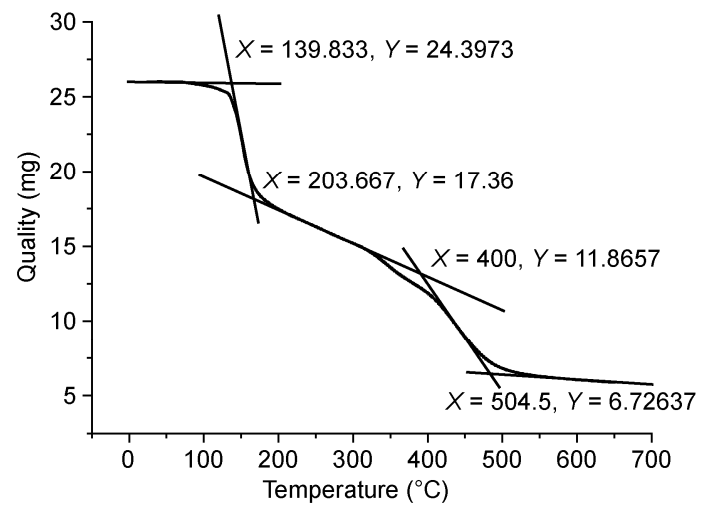

Figure 6 Thermogravimetric analysis of DF2.
In this process, the mass loss ratio was $28.8 \%$, which was caused by the volatility of vinyl ethyl ether. The temperature tolerance of DF2 decomposition $\left(140-200^{\circ} \mathrm{C}\right)$ is much lower than that of positive thermal imaging $\left(250-300^{\circ} \mathrm{C}\right)$. Consequently, DF2 decomposed completely when scanned by an 803-nm laser. DF1, the decomposition product, has good solubility in alkaline media. As a result, the solubility differential between the exposed and unexposed areas in an alkaline developer increased, and this improves the sensitivity of the positive thermal CTP plate [1].

\subsection{Polymer performance}

(1) Possible mechanism of positive thermal CTP plate imaging. It could be concluded from Figure 6 that a possible mechanism for the action of DF2 in positive thermal CTP imaging is as shown in Figure 7.

The DF2 hydroxyl groups were protected by its active ether or ester groups, which could be removed via acidolysis or pyrolysis. So, DF2 is insoluble in alkaline solutions as an inhibitor, but it decomposes into DF1 on exposure to an $830-\mathrm{nm}$ laser, and DF1 is soluble in alkaline media as an accelerator.

(2) Impact of DF2 on alcohol resistance, alkali resistance, sensitivity, and lattice point $(50 \%)$ of positive thermal CTP plate. The imaging performance of a positive thermal CTP plate depends on five main factors: alcohol resistance, alkali resistance, sensitivity, 50\% lattice point, and developing tolerance [15]. Values of the first four parameters are listed in Table 4.

As shown in Table 4, the alcohol resistance and alkali resistance of the plate increased with increasing baking time.

The sensitivity of the plate declined, whereas the alcohol resistance, alkali resistance, and lattice point reducibility
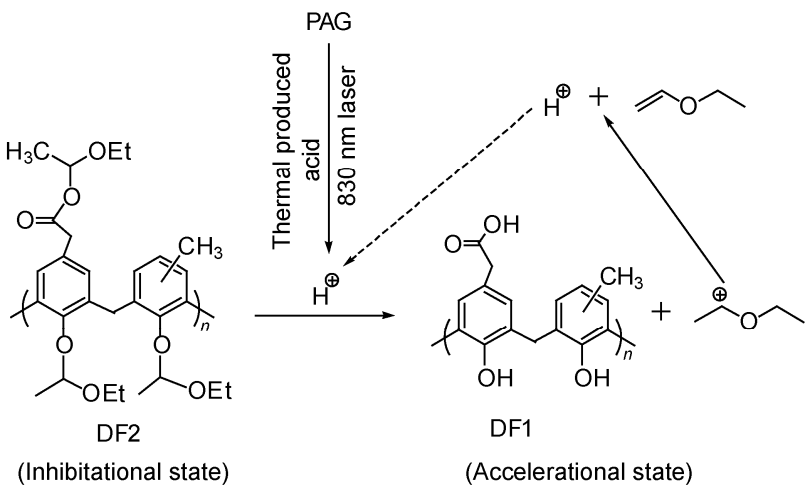

Figure 7 Mechanism of DF2 action in positive thermal CTP imaging.

Table 3 Solubility and melting range

\begin{tabular}{|c|c|c|c|c|c|c|c|}
\hline \multirow{2}{*}{ Type } & \multirow{2}{*}{$\operatorname{MR}\left({ }^{\circ} \mathrm{C}\right)$} & \multicolumn{6}{|c|}{ Solubility (g/100 g) } \\
\hline & & $(1 \%) \mathrm{NaOH}$ & Butanone & Isopropanol & EGMME & PGMME & Toluene \\
\hline DF1 & $133-137$ & 15.8 & 62.3 & 2.5 & 55.2 & 54.6 & 4.8 \\
\hline
\end{tabular}


Table 4 Basic parameters of positive thermal CTP plates

\begin{tabular}{|c|c|c|c|c|c|c|}
\hline Type & $\begin{array}{c}\text { Baking } \\
\text { duration (min) }\end{array}$ & $\begin{array}{l}\text { The mass of } \\
\text { layer }\left(\mathrm{g} \mathrm{m}^{-2}\right)\end{array}$ & $\begin{array}{c}\text { Alcohol- } \\
\text { resistance }(\%)\end{array}$ & $\begin{array}{c}\text { Alkali-resistance } \\
(\%)\end{array}$ & $\begin{array}{c}\text { Sensitivity } \\
(\%)\end{array}$ & $\begin{array}{c}\text { 50\%lattice point } \\
(\%)\end{array}$ \\
\hline \multirow[t]{2}{*}{ CTP-1 } & 10 & 1.61 & 68.9 & 23.1 & 30 & 39.4 \\
\hline & 120 & 1.62 & 77.8 & 30.1 & 35 & 45.1 \\
\hline \multirow{2}{*}{ CTP-2 } & 10 & 1.60 & 72.4 & 34.6 & 40 & 46.8 \\
\hline & 120 & 1.63 & 79.8 & 42.7 & 45 & 47.4 \\
\hline \multirow[t]{2}{*}{ CTP-3 } & 10 & 1.62 & 75.5 & 55.8 & 45 & 46.1 \\
\hline & 120 & 1.60 & 81.6 & 64.7 & 50 & 48.5 \\
\hline \multirow[t]{2}{*}{ CTP-4 } & 10 & 1.62 & 79.6 & 67.2 & 50 & 46.2 \\
\hline & 120 & 1.61 & 85.4 & 76.7 & 55 & 48.1 \\
\hline \multirow[t]{2}{*}{ CTP-5 } & 10 & 1.59 & 74.7 & 72.5 & 50 & 46.8 \\
\hline & 120 & 1.61 & 82.3 & 82.3 & 55 & 48.8 \\
\hline
\end{tabular}

increased with increasing content of DF2 for the same baking time. The alcohol resistance, alkali resistance, and lattice point reproduction of the positive thermal CTP plates were all too low to meet the needs of practical printing when the content of DF2 was below $10 \%$.

Figure 8 shows the patterns for CTP-5. These were obtained using a microscope with a polarized light source. After exposure to IR irradiation (830-nm laser), the plate was developed in alkaline solution and baked for $10 \mathrm{~min}$ (a) or $2 \mathrm{~h}$ (b). The squares in the patterns are regular and have smooth edges. There is no loss from the imaging layer of the unexposed area. The blank part, after exposure and developing, is clean. The two measured values of the $50 \%$ lattice point, obtained using X-Rite iCplates, are $47.1 \%$ and $48.8 \%$. Theoretically, the pattern of the $50 \%$ lattice point is square, and the area in the exposed area is equal to that in the unexposed area, accounting for $50 \%$ each. However, a newly produced positive thermal CTP plate needs a certain period of time to achieve maturation and stability, during which the lattice point of the plates will rise correspondingly, so it is best to keep the measured value for the $50 \%$ lattice point of the newly produced positive thermal CTP plate in the range $46 \%-48 \%$ so that the final value is $48 \%-50 \%$. It is concluded from comparisons of alcohol resistance, alkali resistance, sensitivity, and lattice point reducibility that the CTP-5 plate is the best in terms of all-round imaging performance.

(3) Impact of DF2 on developing tolerance of positive thermal CTP plate. It is necessary for an excellent positive thermal CTP plate to have a wide developing tolerance. According to the results in Table 4, the CTP-5 plate has the best imaging performance. We tested the concentration developing tolerance (Table 5), temperature developing tolerance (Table 6), and time developing tolerance (Table 7) for CTP-5.

The parameters in Table 5 were obtained after developing for $30 \mathrm{~s}$ at $23^{\circ} \mathrm{C}$. It was concluded that the lattice point and sensitivity were still satisfactory even after doubling the
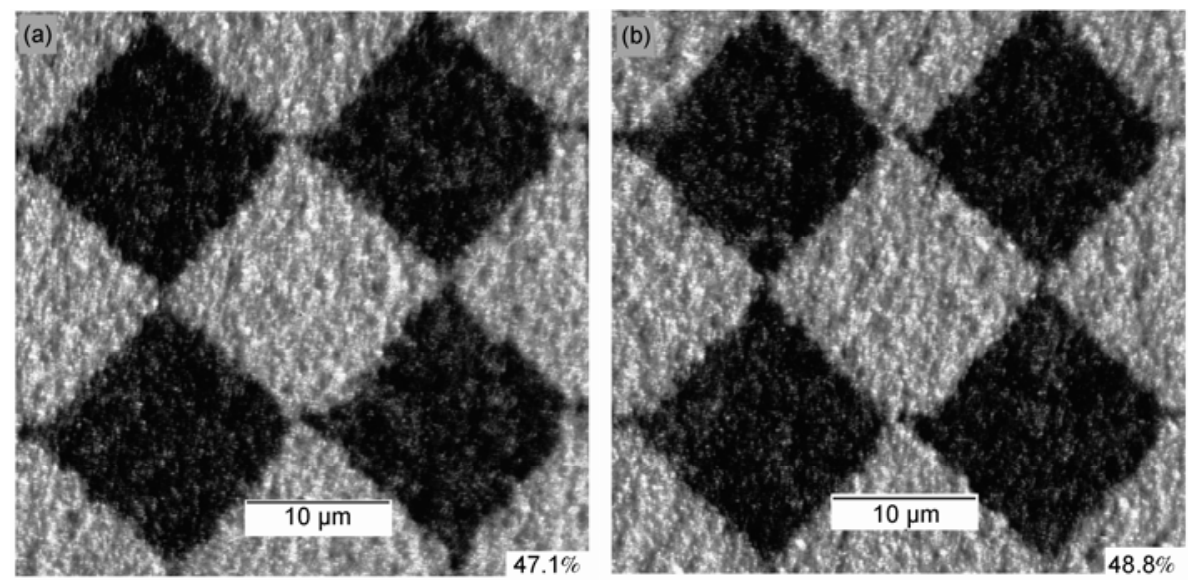

Figure 8 Images of 50\% lattice point of thermal CTP plate baked for (a) $10 \mathrm{~min}$ and (b) $2 \mathrm{~h}$.

Table 5 Developing tolerance of concentration

\begin{tabular}{|c|c|c|c|c|c|c|c|c|}
\hline \multirow{2}{*}{ Chroma } & \multirow{2}{*}{ Sensitivity (\%) } & \multirow{2}{*}{ Density } & \multicolumn{6}{|c|}{ The area of lattice points } \\
\hline & & & $1 \%$ & $2 \%$ & $10 \%$ & $50 \%$ & $98 \%$ & $99 \%$ \\
\hline Undiluted & 50 & 1.23 & 0.9 & 1.6 & 8.7 & 47.8 & 97.4 & 98.2 \\
\hline Dilute & 60 & 1.25 & 1.0 & 1.8 & 8.9 & 48.7 & 97.9 & 98.9 \\
\hline
\end{tabular}


Table 6 Developing tolerance of temperature

\begin{tabular}{|c|c|c|c|c|c|c|c|c|}
\hline \multirow{2}{*}{ Temperature $\left({ }^{\circ} \mathrm{C}\right)$} & \multirow{2}{*}{ Sensitivity (\%) } & \multirow{2}{*}{ Density } & \multicolumn{6}{|c|}{ The area of lattice points } \\
\hline & & & $1 \%$ & $2 \%$ & $10 \%$ & $50 \%$ & $98 \%$ & $99 \%$ \\
\hline 21 & 55 & 1.24 & 1.0 & 1.8 & 8.9 & 49.2 & 97.9 & 98.9 \\
\hline 23 & 50 & 1.23 & 0.9 & 1.6 & 8.7 & 47.8 & 97.4 & 98.2 \\
\hline 25 & 50 & 1.22 & 0.8 & 1.4 & 8.2 & 46.9 & 97.1 & 98.0 \\
\hline 27 & 45 & 1.20 & 0.6 & 1.3 & 7.9 & 46.1 & 96.7 & 98.0 \\
\hline 29 & 35 & 0.92 & 0.3 & 0.8 & 6.8 & 43.8 & 95.6 & 97.1 \\
\hline
\end{tabular}

Table 7 Developing time tolerance

\begin{tabular}{|c|c|c|c|c|c|c|c|c|}
\hline \multirow{2}{*}{ Time (s) } & \multirow{2}{*}{ Sensitivity (\%) } & \multirow{2}{*}{ Density } & \multicolumn{6}{|c|}{ The area of lattice points } \\
\hline & & & $1 \%$ & $2 \%$ & $10 \%$ & $50 \%$ & $98 \%$ & $99 \%$ \\
\hline 20 & 55 & 1.24 & 1.0 & 1.7 & 8.6 & 48.1 & 97.8 & 98.6 \\
\hline 30 & 50 & 1.23 & 0.9 & 1.6 & 8.7 & 47.8 & 97.4 & 98.2 \\
\hline 40 & 50 & 1.23 & 0.8 & 1.4 & 8.2 & 46.7 & 97.3 & 98.1 \\
\hline 50 & 45 & 1.22 & 0.6 & 1.2 & 7.9 & 46.0 & 96.4 & 97.6 \\
\hline
\end{tabular}

dilution of the developer.

The parameters for CTP-5 developed in undiluted developer at different temperatures for $30 \mathrm{~s}$ after exposure are shown in Table 6. With increasing temperature, the lattice point and the density of the unexposed area become lower. When the temperature was above $27^{\circ} \mathrm{C}$, the lattice point reduction was serious. It can be concluded that the temperature developing tolerance should be between 21 and $27^{\circ} \mathrm{C}$.

The parameters for CTP-5 developed in undiluted developer at $23^{\circ} \mathrm{C}$ for different times after exposure are shown in Table 7. The lattice point reducibility of CTP-5 is good and the differences among the values of the densities of the unexposed areas are small. It can be concluded that the tolerance in the developing time is good.

\section{Conclusions}

In conclusion, a novel ester ether resin was synthesized for use in positive thermal CTP imaging as an inhibitor/accelerator. Compared with previously reported inhibitors/ accelerators, DF2 can enhance the solubility differential between the exposed and unexposed areas in alkaline developers. It had better chemical resistance and its decomposition temperature range was suitable for 830-nm laser thermal imaging. Consequently, it can greatly improve the performance of the positive thermal CTP plate.

This work was supported by the City Orient Industrial Company and the Beijing Natural Science Foundation (2112020).

1 Wang $X$. The preparation, properties and imaging study of triazine acid generator and new benzoxazine dissolution inhibitor for thermal sensitive CTP plate (in Chinese). Dissertation for the Master's Degree. Beijing: Beijing Normal University, 2005
2 Celin S, Ken-ichi S, Hans-Joachim T, et al. Thermal digital lithographic printing plate. US Patent. US6534238, 2003-03-18

3 Yu S X, Zou Y Q, Shi Z X. Synthesis of ester ether compound with high acidolysis activity (in Chinese). China Patent. CN01123686.8, 2002-05-08

4 Yu S X, Wang X F, Gu J N, et al. Synthesis of novolac resin-based ester-ether or ether derivatives with high acidolysis activity (in Chinese). China Patent. CN02117827.5, 2002-5-22

5 Yu S X, Cong P J, Yang J R, et al. Molecular design of ethers with high acidolysis activity used for dissolution inhibitor/accelerator in photo/ thermal imaging (in Chinese). Imag Sci Photochem, 2010, 28: 37-46

6 Feng L, Niu H Y, Li M S, et al. Photosensitive composition and its application in positive heat-sensitive CTP plate (in Chinese). China Patent. CN201110451144, 2011-12-30

7 Yeh C Y, Yang M H, Lin M S, et al. Radiation sensitive composition. European Patent. EP201013376, 2010-10-06

8 Li Y, Cheng L, Wang L Y. Chemically amplified photographic materials based on acidolytic polymethacrylate and their applications (in Chinese). Image Technol, 2009, 21: 13-16

9 Sha X Z, Zou Y Q. A new inhibitational/accelerational phenolic etherified resin was synthesized and characterized (in Chinese). Thermos Resin, 2008, 23: 23-27

10 Chemical Industry Standard of the People's Republic of China. Infrared cyanine dye for $830 \mathrm{~nm}$ thermal CTP plates (in Chinese). HG/T 4244-2011, 2011-12-20

11 Zou Y Q, Sha X Z. Carboxy-containing novolac resin and its synthesis method (in Chinese). China Patent. CN200910236586.0, 2010-04-21

12 Zou Y Q, Sha X Z. Process for producing carboxyl novolac resin labile ester and etherification compound (in Chinese). China Patent. CN200910236587.5, 2010-04-14

13 Sha X Z, Zou Y Q. Characteristic of a new inhibitational/accelerational novolac resin used to the thermosensitive CTP performance (in Chinese). Imag Sci Photochem, 2006, 26: 327-333

14 Chemical Industry Standard of the People's Republic of China. Photosensitive emulsion for positive thermal CTP plate (in Chinese). HG/T 4245-2011, 2011-12-20

15 Chemical Industry Standard of the People's Republic of China. Positive thermal CTP plate (in Chinese). HG/T 3804-2006, 2009-06-08

16 Gallucci R R, Going R C. Reaction of hemiacetal esters, acetals, and acylals with alcohols or acetic acid. J Org Chem, 1982, 47: 3517-3521

17 Wang B, Li M Y, Ji S S. Solvent-free tetrahydropyranylation of alcohols with sulfamic acid as reusable catalyst. Synth Commun, 2003, 33: $3929-3934$

Open Access This article is distributed under the terms of the Creative Commons Attribution License which permits any use, distribution, and reproduction in any medium, provided the original author(s) and source are credited. 